\title{
Nukleare Technopolitik in der BRD - zwischen technischer Utopie und sozialer Dystopie
}

Lutz Mez, Daniel Häfner

\section{Zusammenfassung}

Die Atomspaltung trat mit einem Donnerschlag in das Bewusstsein der gesamten Menschheit - und mit einer sechsstelligen Zahl von Toten. Dieser Einstieg ins Atomzeitalter war politisch und technisch irreversibel. Die Probleme der "friedlichen Nutzung" der Kernenergie waren von Anfang an bekannt und wurden auch offen kommuniziert. Je konkreter die Projekte und auch die Interessen der beteiligten Akteure wurden, desto mehr wurden diese Argumente aus dem Diskurs verbannt. In einem historischen Überblick zeigen die Autoren die technischen und sozialen Entwicklungen der Nutzung der Kernenergie auf. Im Kern geht es darum, dass die Atomenergieauch-immer ein staatliches/politisches Projekt war, welches aufdie korrelierenden Interessen von Teilen der Wirtschaft traf. Diese Interessen waren nur in einem »starken Atomstaat umzusetzen; sobald Mitsprache, bspw. in Umweltverträglichkeitsprüfungen, ermöglicht wurde, waren die nuklearen Projekte nicht mehr durchzuführen. Der »Atomstaat« muss nun, am Ende der Nutzung der Kernenergie, durch Partizipationsangebote sehr »weich « agieren, um ein Endlagerprojekt umzusetzen. ${ }^{1}$

\section{Einleitung}

Die Atomspaltung trat mit einem Donnerschlag in das Bewusstsein der gesamten Menschheit - und mit einer sechsstelligen Zahl von Toten. Dieser Einstieg ins Atomzeitalter war politisch und technisch irreversibel. Auf beiden Ebenen wurde versucht, die gewaltigen Mengen Energie einzuhegen, auf der politischen Ebene durch den Versuch der Nicht-Verbreitung von Atomwaffen, auf der technologischen Ebene durch technologische Beherrschung (Klaus/Porst 1949: 7f.). Deshalb hatte die Rede Atoms 
for Peace- Atome für den Frieden - des US-amerikanischen Präsidenten Dwight D. Eisenhower, die er im Dezember 1953 vor der UN-Vollversammlung hielt, auch in Deutschland aufmerksame Zuhörer*innen. Mit der zivilen, also der nicht-militärischen, Nutzung der Atomkraft, sollten die Energieprobleme der Welt gelöst werden. Gleichzeitig sollte aber auch das Bild der Atomspaltung, die mit den Atombomben auf Hiroshima und Nagasaki ins Blickfeld der Menschheit getreten war, umgedeutet werden. Die Spaltung des Atoms für zivile Zwecke sollte billige und zuverlässige Energie liefern, neue Antriebssysteme bspw. für Schiffe ermöglichen sowie zur Bekämpfung von Krankheitskeimen anwendbar sein - und somit Wohlstand für alle schaffen. Die Entwicklung der Atomtechnologien schien schicksalhaft und unaufhaltsam (Radkau 2017: 141).

In der technischen Utopie wurde von vier Phasen der Entwicklung der Atomtechnologien ausgegangen. In der ersten Phase wurden die Schwerwasser- und LeichtwasserReaktoren entwickelt, wobei letztere die heute dominierenden Reaktorlinien darstellen. In der zweiten Phase wurde auf Schnelle Brutreaktoren gesetzt, die im Gegensatz zu den Leichtwasser-Reaktoren nicht mit abgebremsten, sondern unmittelbar mit den bei der Kernspaltung entstehenden »schnellen« Neutronen arbeiten. In der dritten Phase sollte der sogenannte nukleare Brennstoffkreislauf mit Wiederaufarbeitungsanlagen geschlossen und in der vierten Phase sollten Kernfusionskraftwerke errichtet werden. Doch während des Kalten Krieges wurde der nukleare Traum von der »strahlenden $\mathrm{Zu}$ kunft « - befördert durch das nukleare Wettrüsten und die drohende Gefahr eines Atomkrieges - immer stärker zum Alptraum für viele Menschen, die sich daraufhin gegen die Atomkraft engagierten. Zugleich wuchs die Erkenntnis, dass friedliche und militärische Nutzung der Atomenergie nicht voneinander zu trennen sind. Aus der politischen und militärischen Strategie wurde eine »soziale Tatsache« (Blowers 2017).

In den 1970er Jahren entstand mit dem Widerstand gegen Atomkraftwerke (AKW) und unter dem Motto »Atomkraft - Nein danke!« die Anti-Atombewegung, die den generellen Ausstieg aus der Atomindustrie und eine Energiewende forderte. Die Risiken der Großtechnologie für die Menschen und der Atomausstieg gelangten auf die Tagesordnung. In der Folge verschoben sich die soziotechnischen Debatten vom Fortschrittsdenken und der Utopie der Eliten zu Technikkritik und der Realität der (potentiell) betroffenen Bevölkerung. Das Gegenbild zur positiven Utopie - die Dystopie - war von Günter Anders bereits beim Abwurf der Atombomben auf Hiroshima und Nagasaki im August 1945 entworfen worden. Anders hatte verstanden, dass es nun möglich war, das gesamte Leben auf der Erde auszulöschen (Anders 1956). Zusammen mit Robert Jungk wurde er einer der Initiatoren der Internationalen Bewegung gegen Atomwaffen (zu Robert Jungk siehe auch den Beitrag Brunnengräbers »Vom starken zum weichen Atom-Staat« in diesem Band).

Robert Jungk stellte sehr früh den Zusammenhang von Technikoptionen und sozialen Verhältnissen her und prägte hierfür den Begriff »Atomstaat«. Wenn die Atomanlagen völlig geschützt werden sollten, "so wäre die unausweichliche Folge ein Leben voll Verboten, Überprüfungen und Zwängen, die in der Größe, der unbedingt zu vermeidenden Gefahren ihre Rechtfertigung suchen würden« (Jungk 1977: X). »Mit der technischen Nutzbarmachung der Kernspaltung wurde der Sprung in eine ganz neue Dimension der Gewalt gewagt. Zuerst richtete sie sich nur gegen militärische Gegner. 
Heute gefährdet sie die eigenen Bürger. Denn >Atome für den Frieden unterscheiden sich prinzipiell nicht von >Atomen für den Krieg‘. Die erklärte Absicht, sie nur zu konstruktiven Zwecken zu benutzen, ändert nichts an dem lebensfeindlichen Charakter der neuen Energie« (Jungk 1977: IX). Der Einstieg in die Atomenergie ist nach Jungk grundsätzlich irreversibel und würde eine Überwachung und Kontrolle der Entsorgungslager notwendig machen und das politische Klima so noch lange Zeit beeinflussen (ebd. XVIII). Bis zum heutigen Zeitpunkt hat Jungk mit dieser Aussage recht: es wird noch Jahrzehnte dauern, mit den »Ewigkeitslasten« (Brunnengräber 2019) der Nutzung der Atomenergie kontrolliert umzugehen.

Im folgenden Beitrag wollen wir zunächst die Geschichte der Atomenergie in der Bundesrepublik Deutschland ${ }^{2}$ auf der Grundlage von verschiedenen Entwicklungsphasen skizzieren: (1.) Utopische Vorstelllungen, (2.) Atomforschung und Entwicklung, (3.) Atompolitik und Atomprogramme, (4.) Anti-Atom-Proteste, Energiewende und Atomausstieg. Dabei wollen wir folgendes zeigen: a) die technischen Risiken und Gefahren der Nutzung der Atomkraft (wie auch die Entsorgungsprobleme) waren von Anbeginn bekannt; b) die kommerziellen und politischen Interessen bestimmten, wie Vorteile und Nachteile der Atomenergie kommuniziert wurden; c) diese Interessen führten zur staatlichen Unterstützung der Atomtechnik auf allen Ebenen; und d) nach den SuperGAUs in Tschernobyl und Fukushima begann die atompolitische Wende.

\section{Atomkraft - die Utopie von der strahlenden Zukunft}

Die Atomkraft war von Anfang an von einem Mythos und einer Aura von Macht, Stärke und Fortschritt umgeben (Radkau 1983: 92). In den 1950er Jahren war die Nutzung der Atomtechnologien deshalb nicht nur eine Utopie von Konservativen oder Rechten, sondern auch integraler Bestandteil linker und progressiver Utopien. Auch der Staatssozialismus des Ostblocks setzte konsequent auf die Spaltung des Atoms und der weltweit erste Reaktor zur kommerziellen zivilen Stromproduktion wurde 1954 in Obninsk $^{3}$ bei Moskau in Betrieb genommen. Die Nukleartechnik mit scheinbar unbegrenzter, von natürlichen Ressourcen nahezu unabhängiger Energieproduktion passte perfekt zur Utopie der Entfesselung der Produktivkräfte auf dem Weg ins kommunistische Paradies. Ernst Bloch schrieb in seinem Hauptwerk »Das Prinzip Hoffnung «" ${ }^{4}$ die Atomenergie schaffe »aus Wüste Fruchtland, aus Eis Frühling. Einige hundert Pfund Uranium und Thorium würden ausreichen, die Sahara und die Wüste Gobi verschwinden zu lassen, Sibirien und Nordamerika, Grönland und die Antarktis zur Riviera zu verwandeln«(Bloch 1959: 775). Bereich der Atomenergie engagiert, bspw. sollte in Stendal das größte AKW der Welt entstehen. Der Reaktor vom Typ AM-1 (Abkürzung für »Friedliches Atom«) hatte eine elektrische NettoLeistung von $5 \mathrm{MW}$ und eine thermische Leistung von $30 \mathrm{MW}$. 
Deutlich weniger naiv-utopisch schreibt Edgar Salin in seiner »Ökonomik der Atomkraft« (1955: 6):

»Auch bei solchen Überlegungen muss eine Warnung vor utopischen Hoffnungen und eine Warnung vor allzu sicheren Berechnungen an der Spitze stehen. So wenig wie die Eisenbahn, die Elektrizität, das Flugzeug, auf die man solche Hoffnungen setzte, wird die Atomenergie das Friedensreich heraufführen.«

Salin ist aber sicher, dass die Atomenergie die Wirklichkeit von morgen darstelle und beschreibt drei Dimensionen der Atomkraft, die später noch diskursiv zum Tragen kommen sollten. Zunächst übernimmt er amerikanische Berechnungen, wonach die Elektroenergie - in einem Schnellen Brüter erzeugt - lediglich variable Kosten von 0,0056 Pfennig pro Kilowattstunde haben solle (Salin 1955: 30). Damit wäre die Elektroenergie too cheap to meter. Das Sicherheitsbedürfnis der Bevölkerung in Bezug auf die Atomenergie gäbe aber »den wenigen, die am Schalthebel der neuen Kräfte sitzen, eine ungeheure Macht über die Massen - es droht die Gefahr einer Technokratie von solcher Machtfülle, dass alle Diktaturen der Vergangenheit daneben als bloße Stümperei erscheinen « (ebd.: 41). Er räumt auch ein, dass die Atommüllfässer auch am tiefsten Meeresgrund »ihre unheilvollen Strahlen senden« (ebd. 1955: 41).

Mit diesem Verweis auf den problematischen Umgang mit radioaktiven Abfällen ist Salin in der Fachwelt nicht allein, so widmet die Zeitschrift "Atompraxis« der Entsorgungsfrage bereits 1956 einen kleinen Sonderteil. Dort bemerkte H.A. Künkel:

»Es werden ja immer wieder warnende Stimmen laut, dass bei zunehmender Verwendung der Atomenergie [...] keine Möglichkeit mehr für die gefahrlose Abfallbeseitigung mehr bestünde und wir in weiterer Zukunft [...] rechnen müssten, mit allen bekannten Folgeerscheinungen der gesundheitlichen und genetischen Schäden für das Menschengeschlecht« (Künkel 1956: 273).

Im Rahmen des technischen Fortschrittglaubens wird aber auf zukünftige Technologien zur Lösung der Problematik verwiesen, bspw. auch die Verwendung der Abfälle in Ton gebunden als »Wärmesteine« in Haushalten (Graul 1956: 271f.).

So verfing die positive technische Utopie auch im politischen Raum. Die westeuropäische Sozialdemokratie setzte dabei insgesamt früher und konsequenter auf das Atom als konkurrierende konservative Parteien. Der technologiepolitische Vordenker der SPD, Leo Brandt, bezeichnete die Atomkraft als »eines der kostbarsten Geschenke, das die Natur für den Menschen bereithält«, mit dem man zum Beispiel die Kultivierung der Urwälder anpacken könne. Auf einer DGB-Kundgebung wurde die neue Energie als »Geschenk des Himmels« bezeichnet, welches »im richtigen Moment zu Hilfe kommt«. Die Atomkraft sollte die Säule der zweiten industriellen Revolution bilden. »Die Atomenergie kann zu einem Segen für Hunderte von Millionen Menschen werden, die noch im Schatten leben«, heißt es im Atomplan der SPD, der 1956 auf dem Münchner Parteitag verabschiedet wurde. Und in der Präambel des Grundsatzprogramms von 1959 wird die Nutzung der »Urkraft des Atoms« mit der Erwartung verknüpft, »daß der Mensch im atomaren Zeitalter sein Leben erleichtern, von Sorgen befreien und Wohlstand für alle schaffen kann«. 
Konrad Adenauer war bis zum Ende seiner Kanzlerschaft kein überzeugter Anhänger der Nutzung der Atomenergie. Bei der Atomkernspaltung ging es ihm und Franz Josef Strauß vor allem um die militärische Option. Und da die zivile Nutzung dazu nützlich sein konnte, wurde 1955 ein Bundesministerium für Atomfragen (BMAt) - mit Franz Josef Strauß an der Spitze - gegründet. Sein Parteifreund Balke, der ihn ein Jahr später ablöste, propagierte: »Wer keine Atomkraft im Angebot hat, der wird auch keine Staubsauger mehr verkaufen«. Die Angst um die technologische Vorherrschaft der Siegermächte des Zweiten Weltkriegs überwog gegenüber der Frage der sozialen Bedingungen der Nukleartechnik, die für den Betrieb der Atomanlagen und für die Entsorgung des Atommülls geschaffen werden mussten. Risiken und soziotechnische Folgen der Atomtechnik wurden zwar fachöffentlich relativ offen diskutiert, aber kaum gesellschaftlich debattiert. Im Vordergrund standen wirtschaftliche Motive und die militärische Option, von denen auch die deutsche Atompolitik von Anfang an geleitet wurde.

\section{Atomforschung und Entstehung der bundesdeutschen Atomindustrie}

Wegen der alliierten Verbote, die den Verlierern des Zweiten Weltkriegs jegliche Tätigkeit auf dem Gebiet der Atomenergie - militärisch und zivil - untersagten, konnte die Bundesrepublik erst nach dem Inkrafttreten der Pariser Verträge 1955 offiziell mit der »friedlichen Atomforschung und -technik beginnen. Halboffizielle Initiativen gab es jedoch schon vorher: (1.) die »Kernphysikalische Kommission« des Deutschen Forschungsrates, (2.) die »Kommission für Atomphysik« der Deutschen Forschungsgemeinschaft (ab 1952), (3.) den Lehrstuhl für Strahlen- und Kernphysik an der Universität Bonn (ab 1953). Außerdem hatten deutsche Kernphysiker die Möglichkeit, in supranationalen Organisationen wie der European Organization for Nuclear Research $\left(\mathrm{CERN}^{5}\right) \mathrm{zu}$ arbeiten. Am Göttinger Max-Planck-Institut konzipierte die »Reaktorphysikalische Arbeitsgruppe« einen Forschungsreaktor. Zur Finanzierung dieses Vorhabens erfolgte im November 1954 auf Initiative einiger Industrievertreter die Gründung der »Physikalischen Studiengesellschaft $\mathrm{mbH}$ «, an der sich 16 - später wurden es fast 30 bundesdeutsche Konzerne der Elektro-, Chemie-, Maschinenbau- und Metallindustrie beteiligten $^{6}$; sie bildeten den Kern der späteren Atomindustrie in der Bundesrepublik Deutschland (Mez 1979: 29f).

Dieser Konsens - der beinahe als Pro-Atomhysterie beschrieben werden kann - galt insbesondere innerhalb der Eliten. In der breiten Bevölkerung waren im Jahr 1959 nur $8 \%$ vorbehaltlos für die Kernenergie, während $17 \%$ befürchteten, diese würde in den Atomkrieg führen (Radkau 2017: 143). Und so regte sich bereits gegen den ersten Forschungsreaktor Widerstand in der lokalen Bevölkerung in den Hardtgemeinden (ehemalige selbständige Gemeinden bei Karlsruhe), die das zusätzliche Risiko der Kerntech- 
nologie nicht hinnehmen wollte. Der Protest wurde aber befriedet und war insbesondere deshalb nicht erfolgreich, weil der lokale Protest der »Fortschrittsfeinde im Atomzeitalter« auf überregionaler und nationalstaatlicher Ebene gegen das Fortschrittsparadigma weder verstanden noch unterstützt wurde (Gleitsmann/Oetzel 2012: 144). Im Gegenteil, die Protestierenden wurden als »kommunistisch unterwandert « und als »Radaubrüder ( phobie« (Graul 1957: 468).

Als im Mai 1955 das Verbot der Alliierten aufgehoben wurde, einigten sich Staat, Wirtschaft und Wissenschaft auf ein enges gemeinsames Vorgehen, um den Entwicklungsrückstand gegenüber dem Ausland aufzuholen. Die Industrie sicherte sich den Haupteinfluss auf die kommerzielle Nutzung, während dem Staat die Hauptinitiative bei der Finanzierung und der Strahlenschutzgesetzgebung übernahm. Zur Unterstützung des Aufbaus der Atomindustrie wurde im Oktober 1955 das BMAt gegründet, das aber mit sehr geringen personellen Ressourcen keine Kontroll- oder Steuerungsfunktionen ausüben konnte - und auch nicht sollte. Der erste Atomminister Franz Josef Strauß erläuterte bei der Konstituierung der Deutschen Atomkommission am 26.1.1956: »Mit den Pariser Verträgen haben wir auf die Herstellung von Atomwaffen im Gebiete der Bundesrepublik Deutschland verzichtet, aber freie Hand auf friedlichem Gebiete bekommen. Wir sind uns der Tatsache bewusst, dass wir einen 10-15jährigen Rückstand gegenüber USA, Großbritannien, der Sowjetunion und anderen Ländern aufzuholen haben« (Strauß 1956).

Im August 1955 hatte in Genf die UNO-Konferenz zur friedlichen Nutzung der Atomenergie stattgefunden, auf der die deutschen Delegierten die Dringlichkeit eines nuklearen internationalen Standards betonten (Keck 1984: 52-56). Die deutsche Atompolitik zielte auf die Entwicklung eines nationalen Nuklearsektors ab, also nicht nur auf Produktion und Betrieb von Atomkraftwerken (AKW), sondern auch auf alle anderen Anlagen des nuklearen Brennstoffkreislaufs - von der Urangewinnung über die Anreicherung bis hin zur Wiederaufarbeitung und »Entsorgung« des Atommülls. Ursprünglich galt das Atomprogramm als integraler Bestandteil einer allgemeinen Industriepolitik zur Wiederherstellung einer lebensfähigen Wirtschaft auf den internationalen Märkten, zusammengefasst als das sogenannte »Modell Deutschland «. ${ }^{7}$

Die deutsche Atomindustrie bestand aus Unternehmen der Elektroindustrie, der Maschinenbauindustrie und der Chemischen Industrie. Diese Unternehmen hatten die Mehrheit in der »Deutschen Atomkommission« $(\mathrm{DAtK})^{8}$, dem Hauptberatungsgremium des Bundesministeriums für Atomfragen und zugleich dem Zentrum der Atom-

7 Helmut Schmidt sagte im Wahlkampf 1976: Das »Modell Deutschland « zeichnet sich dadurch aus, dass alle anderen Länder die üblichen drei Produktionsfaktoren haben, nämlich Arbeit, Kapital und Boden - wir in Deutschland haben aber vier davon. Der vierte Produktionsfaktor ist der soziale Frieden. Siehe https://www.diw.de/documents/dokumentenarchiv/17/39481/offe.pdf und https://de .wikipedia.org/wiki/Helmut_Schmidt, zuletzt geprüft am 11.11.2020.

8 Unter dem Vorsitz des Ministers für Atomfragen gehörten der Kommission 27 Personen an, die aus Wissenschaft, Technik, Wirtschaft und den Cewerkschaften kamen. Zu gleichberechtigten Vizepräsidenten wurden Otto Hahn, Staatssekretär Leo Brandt und Karl Winnacker ernannt. Die Atomkommission berief fünf weitere Fachkommissionen ein, die sich mit einzelnen Aufgaben befassten. Zeitweilig gehörten der DAtK über 200 Mitglieder an. 
politik ${ }^{9}$ (Keck 184: 56f). Alle an der Entwicklung der Atomenergie und dem Aufbau der Atomindustrie interessierten Wirtschaftskreise sowie Vertreter von Wissenschaft und Staatsadministration waren hier versammelt. Schlüsselpositionen besetzten die im Aufsichtsrat der Physikalischen Studiengesellschaft $\mathrm{mbH}$ vertretenen Großkonzerne und sicherten der Industrie so den entscheidenden Einfluss (Mez 1979: 30). Die Konstruktion eines kleinen Fachministeriums bei gleichzeitiger Bildung eines interessengebundenen Beratungsapparates führte praktisch zur uneingeschränkten Kontrolle der Atomkommission über die Atompolitik.

\section{Die Atomprogramme 1957 - 1976}

Die frühe Atomkraft-Ära war von einem enormen technologischen Optimismus geprägt, der bis in die 1970er Jahre auf einem allgemeinen politischen Konsens zwischen allen im Parlament vertretenen Parteien beruhte. Eine umfangreiche finanzielle Unterstützung der öffentlich-privaten nuklearen Forschung und Entwicklung für Produzenten und Betreiberunternehmen einzelner Projekte stand im Mittelpunkt der deutschen Atomprogramme. ${ }^{10}$ 1956-1957 startete die DAtK das erste offizielle deutsche Atomprogramm, auch »Eltviller-Programm« genannt (Prüß 1974: 70). Bis 1965 sollten parallel fünf $100 \mathrm{MW}$ Atomreaktoren entwickelt werden. Die Anzahl fünf kam nicht aus technischen Erwägungen zustande, sondern war auf die Zahl der konkurrierenden Unternehmenskonsortien zurückzuführen, die an der Durchführung des Programms beteiligt waren. Alle Teilnehmenden wollten einen Anteil an der finanziellen Unterstützung der deutschen Regierung haben. Aber nur zwei der fünf geplanten Reaktoren wurden gebaut, ${ }^{11}$ und bereits 1960 wurde das Eltviller Programm durch ein »Programm für fortgeschrittene Reaktoren und Versuchsatomkraftwerke« modifiziert (Mez 1979: 31).

Im Dezember 1959 wurde das erste bundesweite Atomgesetz (AtG), das »Gesetz über die friedliche Verwendung der Kernenergie und den Schutz gegen ihre Gefahren « verabschiedet. Bis dahin hatten Gesetze und Verordnungen der Länder die wirtschaftlichen und gesundheitlichen Interessen in Bezug auf Strahlung, Kernkraft sowie die Herstellung von Isotopen und deren Verwendung geregelt. Trotz des teilweisen Scheiterns des ersten Programms setzte sich der Konsens innerhalb der Eliten fort. Siemens begann auf der Basis einer Westinghouse-Lizenz mit dem Bau von Druckwasserreaktoren (DWR), und AEG, ein Tochterunternehmen von General Electric, begann mit dem Bau von Siedewasserreaktoren (SWR). Parallel zum Eltviller Programm bestellte RWE,

Die DAtK bestand von 1956 bis 1971. Aus einem der Arbeitskreise der Fachkommission IV Strahlenschutz entstand später die Strahlenschutzkommission.

10 Im Rahmen der vier Atomprogramme von 1957-1976 wurden Forschungsmittel des Bundesministeriums für Forschung und Technologie (BMFT) in Höhe von insgesamt 10 Mrd. € ausgegeben. Dieser finanzielle Beitrag war eine der Voraussetzungen für den Erfolg der Atomindustrie, da viele private Investoren in den späten 1950er Jahren aufgrund niedriger Rentabilitätserwartungen AKW ablehnten (Radkau 1983: 196).

11 Der 15 MW-Forschungsreaktor in Jülich - Inbetriebnahme 1966 und der 100 MW Leistungsreaktor in Niederaichbach, Inbetriebnahme 1972, endgültige Abschaltung 1974. 
damals der größte deutsche Stromversorger, zusammen mit dem Bayernwerk das Versuchsatomkraftwerk Kahl ( $15 \mathrm{MW}_{\mathrm{el}}$ ) von AEG, aber alle Kernkomponenten wurden von General Electric geliefert. Im Jahr 1961 ging das erste bundesdeutsche AKW in Kahl am Main in Betrieb. Die großen Verbundunternehmen RWE und Bayernwerk wollten schnell Betriebserfahrungen mit Atomanlagen machen. 1958 startete AEG in Zusammenarbeit mit General Electric und der Hochtief AG die Planung eines 200-MW AKW für RWE. Der Bau begann Ende 1962 und 1967 wurde das KKW Gundremmingen ans Netz angeschlossen (237 $\left.\mathrm{MW}_{\mathrm{el}}\right)$.

Das 2. Atomprogramm (1963-1967) war das bisher umfassendste Instrument zur Förderung und Koordinierung der Atomenergie. Es integrierte Forschung, Entwicklung, Bau sowie Betrieb von Pilotanlagen und Prototypen. Die Strategie zur Entwicklung von Kernreaktoren knüpfte an die Erfahrungen in den USA an. In einem Nahprogramm ging es um die Übernahme von im Ausland erprobten Technologien und Reaktorkonstruktionen sowie um die Entwicklung des Hochtemperaturreaktors. Die Strategie umfasste auch ein Hilfsprogramm für den Bau und den Strahlenschutz sowie das Fernprogramm zur Entwicklung von nur langfristig realisierbaren »Schnellen Brütern«. Die direkten Subventionen für die Atomindustrie wurden während des zweiten Atomprogramms um den Faktor Fünf gesteigert. Mit Ablauf des Programms hatte Deutschland zu den internationalen Standards der Atomtechnologie aufgeholt. In diesen zeitlichen Kontext gehört auch die Inbetriebnahme des sogenannten Forschungsbergwerks Asse II, welches ab 1965 in einem ehemaligen Salzbergwerk eingerichtet wurde.

Da die staatliche Entwicklungspolitik als erfolgreich beurteilt wurde, ging die Bundesregierung diesen Weg auch im 3. Atomprogramm (1968-1972) weiter. Die staatlichen Subventionen wurden noch einmal enorm gesteigert. Während bis Mitte der 1960er Jahre der größte Teil in die Grundlagenforschung und die Ausbildung in der Fachwissenschaft geflossen war, hatte die Kerntechnik - die kommerzielle Umsetzung der Grundlagen - nun einen Stand erreicht, auf dem sie den Löwenanteil der Mittel verschlang.

Das 4. Atomprogramm ${ }^{12}$ (1973-1976) stand unter dem Zeichen der ersten Ölpreiskrise und dem gemeinsamen Auftreten der erdölexportierenden Länder. Die große Abhängigkeit der deutschen Wirtschaft von importiertem Erdöl zwang die Bundesregierung im Oktober 1973 zur Verabschiedung eines Energieprogramms, das schon ein Jahr später »fortgeschrieben« werden musste. Die Kernenergie wurde in den Mittelpunkt der energiepolitischen Agenda gerückt und die Regierung beschloss, Öl durch ein ehrgeiziges Atomprogramm zu substituieren. Die Atomkraft sollte bis 1985 die höchsten Zuwachsraten aller Energieträger haben. Ihr Anteil am Primärenergieverbrauch sollte von $1 \%$ (1973) auf $15 \%$ (1985) gesteigert werden. Mit dem Neubau von 40 Reaktoren das entspricht einer installierten Leistung von 50.000 MW - sollte der Verteuerung von Mineralöl begegnet werden, Arbeitsplätze geschaffen und die deutsche Energieversorgung unabhängiger von Ölimporten werden.

Nicht einmal die Hälfte der AKW-Leistung, die in den Ausbauplänen von 1974 projektiert waren, wurde wirklich realisiert. Die Ursachen hierfür waren der tendenziell

12 In diesem Programm wurde das Ziel verfolgt, die Kernkraftkapazität bis 1985 um das 20-fache zu erhöhen, d.h. die Gesamtkapazität von 40-50.000 MW und einen Anteil von bis zu $40 \%$ der Stromerzeugung in AKW. Dies wurde 1974 erneut bestätigt (Deutscher Bundestag 1974). 
stagnierende Strombedarf in der Industrie, Überkapazitäten bei der Stromerzeugung und die immer stärker werdenden öffentlichen Kontroversen über die Nutzung der Atomenergie. Im Mai 1977 verabschiedete die Bundesregierung das »Programm Energieforschung und Energietechnologien 1977 bis 1980«. Zwar sollte die nicht-nukleare Energieforschung finanziell erheblich stärker gefördert werden, doch im Atomenergiebereich wurden keine neuen Prioritäten gesetzt: neue Reaktorlinien einschließlich des Schnellen Brüters wurden weiterhin gefördert. Von insgesamt 6,5 Mrd. DM entfielen über $75 \%$ der Gesamtmittel auf die Atomenergie.

Die Bundesrepublik Deutschland war von Anfang an für die Einführung eines nuklearen Brennstoffkreislaufs im eigenen Land. Bereits während des Zweiten Weltkriegs waren Urananreicherungstechnologien entwickelt worden und nach 1955 wurde Deutschland Partner im trinationalen Anreicherungsunternehmen Urenco Ltd. In den 1970er Jahren bauten und betrieben die drei Partner von Urenco Pilot- und Demonstrationsanlagen in Capenhurst, Almelo und Gronau. Gronau wurde 1978 als Standort für die dritte Urananreicherungsanlage ausgewählt. Die Anlage wurde 1985 in Betrieb genommen. Die erste Stufe der jährlichen Trennarbeiten von 1.000 Tonnen Uran wurde 1998 erreicht. Für die derzeit mehr als 100.000 Kubikmeter abgereicherten Urans auf dem Gelände der Urenco sind noch keinerlei Endlagerkapazitäten vorgesehen, bisher wird das Material als Wertstoff $\mathrm{zu}$ weiteren Verwendung deklariert, im Falle einer Umdeklarierung als »Abfall« wäre die aktuelle Endlager-Planung der BRD überfordert.

Zwischen 1971 und 1990 wurde im Kernforschungszentrum in Karlsruhe eine PilotWiederaufarbeitungsanlage (WAK) betrieben, in der rund eine Tonne Plutonium abgetrennt wurde. ${ }^{13}$ Nachdem die Chemische Industrie zu Beginn der 1970er Jahre alle Aktivitäten im Rahmen der deutschen Atomindustrie aufgegeben hatte, übernahmen die Stromversorgungsunternehmen und Betreiber der AKW die Initiative zum Bau und Betrieb einer Wiederaufarbeitungsanlage in Deutschland. Im Jahr 1980 wurden weitere Standorte gesucht. Nachdem der Standort Volkmarsen recht bald aufgegeben wurde, lag der Schwerpunkt der Planung auf der Realisierung einer Wiederaufarbeitungs- und Brennstoffproduktionsanlage in der bayerischen Gemeinde Wackersdorf. Der starke Widerstand der lokalen Bevölkerung, der von der internationalen Anti-Atom-Bewegung unterstützt wurde, führte - neben wirtschaftlichen Gründen - schließlich zum Rückzug der Atomindustrie, um die Wiederaufarbeitungsanlage Wackersdorf 1989 fertigzustellen.

Der Bau der ersten Atommeiler fiel in die Zeit des wirtschaftlichen Aufbruchs der Bundesrepublik. Die zivile Nutzung der Atomkraft wurde in Deutschland mit F\&EProgrammen des Bundes mit Gesamtkosten von über 15,3 Mrd. Euro eingeleitet. Ein Dutzend Jahre nach dem Start der ersten kommerziellen Reaktoren in den USA hatten die deutschen Hersteller mit den Weltmarktstandards gleichgezogen. Insbesondere nach der 1. Ölpreiskrise 1973/74 wurde von der sozialliberalen Koalition der massive Ausbau der Atomindustrie betrieben. Der kommerzielle Betrieb großer Kernkraftwerke fiel zufällig mit der ersten Ölpreiskrise von 1973 zusammen. Das KKW Biblis A wurde

13 Die WAK hatte eine Kapazität von 35 t pro Jahr mit einer Anreicherung bis zu $3 \%$ U-235-Äquivalen t. Bis zum Ende des Betriebs im Jahre 1990 wurden 208 t bestrahlter Kernbrennstoff aufgearbeitet und über $1 \mathrm{t}$ Plutonium abgetrennt. 
1974 in Betrieb genommen - mit einer elektrischen Leistung von 1.200 MW, der damals größte Kernreaktor der Welt. Darüber hinaus hatten die Exportverträge mit Brasilien, Iran und Argentinien zur Entwicklung der Industrie beigetragen. 1975 hatte die KWU Aufträge über 30 Mrd. DM (15 Mrd. €) akquiriert und war die Nummer drei in der weltweiten Atomindustrie.

Damit endete die Phase der Atompolitik, die sich weitgehend auf den Bereich der Industriepolitik beschränkte. Da sich diese auf Forschung und Entwicklung konzentrierte, hatte sie zunächst kaum öffentlich spürbare wirtschaftliche und soziale Auswirkungen. Ein relativ kleiner (elitärer) Kreis aus Wissenschaft, Politik und Industrie stellte auf nationalstaatlicher Ebene einen hegemonialen Konsens in Bezug auf den Fortschritt durch Atomtechnologien her. Angesichts der Ergebnisse könnte man argumentieren, dass die frühe Atompolitik der deutschen Regierung geradlinig und erfolgreich war, was die Unterstützung der Gründung einer deutschen Atomindustrie mit anerkannter - wenn auch nicht einheimischer - technischer Kompetenz angeht. Das Thema der Entsorgung wurde aber weitgehend ausgeklammert. Zum einen galt das Paradigma von disperse und dilute, also das Verdünnen und Freisetzen von radioaktiven Abfällen, zum anderen waren bestimmte »Reststoffe «, insbesondere das Plutonium, erwünscht. In den technisch-utopischen Vorstellungen erschien die Entsorgungsfrage darüber hinaus als marginales Problem. Traf der hegemoniale nationalstaatliche Konsens jedoch in Konkretion der Planungen auf die Bevölkerung, so war in der Durchsetzung auch der "harte« Atomstaat angelegt. Die Vertreter"innen der Ideologie des Fortschritts, gepaart mit militärischen Ambitionen, wollten Protest nicht zulassen.

\section{Anti-Atom-Proteste, Fall der Atomindustrie und Atomausstieg}

In der zweiten Phase der bundesdeutschen Atompolitik ab 1975 gewann das Verhältnis zwischen nationalstaatlichen Akteuren und lokaler Bevölkerung eine neue Dynamik. Diese Phase wurde durch erhebliche öffentliche Proteste gegen Standorte von Atomanlagen ausgelöst. Diese Proteste vernetzten sich relativ schnell überregional und die sich entwickelnde Anti-Atom-Allianz gab das Motto aus: »...und auch nicht anderswo«. Die entstehende Bewegung konnte wissenschaftliche, politische und ideologische Ressourcen mobilisieren, die sie wirkmächtig werden ließ. Die Proteste zeigten Wirkung. Das geplante AKW Wyhl in Baden-Württemberg, wo sich 1975 die Anti-AKW-Bewegung »konstituierte«, musste aufgegeben werden. Im Jahr 1979 musste Ministerpräsident Albrecht von Niedersachsen die Pläne für ein nukleares Entsorgungszentrum in Gorleben als »politisch nicht durchsetzbar« erklären. Und auch das später geplante »Integrierte Entsorgungskonzept« mit räumlich voneinander getrennten Anlagen konnte nicht umgesetzt werden.

1980 schlug eine Enquete-Kommission des Bundestages einen paradigmatischen Wandel in der Energiepolitik weg von der Atomkraft als eines der Szenarien vor (Deutscher Bundestag 1980). Solche Faktoren und Ereignisse trugen zu einer breiten Verschiebung der deutschen öffentlichen Meinung, der Bildung der Partei Die Grünen und schließlich 1983 zur Wahl der Grünen in den Deutschen Bundestag bei (Brand et al. 1986). Dann kam die Atomkatastrophe in Tschernobyl von 1986. Diese Katastrophe 
überstieg bei weitem die Vorstellungen eines GAU, des Größtmöglichen Annehmbaren Unfalls, und löste weltweit eine Debatte über nukleare Gefahren aus. Auch die wirtschaftlichen Risikodimensionen von Kernkraftwerken für eine Volkswirtschaft wurden drastisch verdeutlicht und eine neue Bewertung der Kernenergie in Politik und Industrie angeregt.

In der damaligen Bundesrepublik Deutschland reagierte die Regierung, bestehend aus CDU, CSU und FDP, mit der Einrichtung des Bundesministeriums für Umwelt, Naturschutz und Reaktorsicherheit (BMU). Die Grünen forderten die sofortige Abschaltung aller Atomanlagen. Die SPD beschloss den Atomausstieg innerhalb von zehn Jahren. Darüber hinaus begannen Landesregierungen, Kommunen, Parteien und Gewerkschaften, sich mit der Frage zu beschäftigen, ob der Einsatz der Atomtechnik für die Zukunft sinnvoll und vertretbar sei. Der Abstieg der deutschen Atomwirtschaft hatte begonnen (Radkau/Hahn 2013). Nachdem die Wiederaufarbeitungsoption in Deutschland aufgegeben wurde, versuchte die Atomindustrie seit 1992, mit allen Parteien einen Energiekonsens über einen "politisch ungestörten« Betrieb von Kernkraftwerken zu erzielen. Sie argumentierte, dass der Energiekonsens für die Betreiber sieben Komponenten umfassen sollte: die Definition der regulären Lebensdauer von AKW; die Akzeptanz der Stromerzeugung in großen Kraftwerken; Ausschluss erweiterter Stromeinfuhren; die Aufgabe der Wiederaufarbeitung nach Beendigung bestehender internationaler Verträge; Verarbeitung von Plutonium für MOX-Brennstoff; die Suche nach einer internationalen Alternative für die Endlagerung von hochradioaktiven Abfällen ${ }^{14}$; und die Schaffung von zwei Endlagern für niedrig- und mittelaktiven Atommüll in Deutschland. Im Frühjahr 1993 begannen die Konsensgespräche, aber alle Versuche scheiterten (Mez 1997: 433ff).

Trotz kontinuierlicher und breit angelegter öffentlicher Kritik stellte sich die Atompolitik der Bundesregierung bis 1998 auf die Seite der pro-nuklearen Allianz und unterstützte die Industrie durch eine Reihe von Steuer- und Regulierungsprivilegien. Diese offizielle Position änderte sich nach der Bundestagswahl 1998, als die erste rot-grüne Bundesregierung den Ausstieg aus der Nutzung der Kernenergie ankündigte. Der Koalitionsvertrag zwischen Sozialdemokraten und Grünen enthielt ein spezielles Kapitel $\mathrm{zu}$ diesem Thema. Nach 20 Monaten Verhandlungen zwischen Atomindustrie und Regierung waren eine Reihe von Kontroversen gelöst worden, und Juni 2000 wurde eine Einigung über den schrittweisen Ausstieg aus der Kernenergie erzielt. In der Atompolitik bewirkte die rotgrüne Bundesregierung also eine fundamentale Wende. Ergebnis der mit der Atomwirtschaft getroffenen Vereinbarung war unter anderem, dass die Betriebsgenehmigungen der AKW befristet und der Bau von neuen Reaktoren sowie - ab dem 1. Juli 2005 - Transporte von abgebrannten nuklearen Brennelementen zur Wiederaufarbeitung verboten wurden. Damit war die Festlegung auf die direkte Endlagerung verbunden und erstmals setzte ein großes Industrieland wie Deutschland ein klares Zeichen in der Atompolitik. Die Vereinbarung zum Atomausstieg führte zur Novellierung des Atomgesetzes. Statt der Förderung der Kernenergie war nunmehr ihre geordnete Beendigung Zweck des Gesetzes. Für jeden Reaktor wurde eine Restlaufzeit

14 Entsprechende Verhandlungen führte die Nukem bereits im Jahr 1976 mit der UdSSR und ab 1983 im der VR China - die allerdings scheiterten (Stephany 2005: 74f.). 
festgelegt, nach deren Ablauf die Betriebsgenehmigung erlischt. Ferner wurden die Betreiber zum Bau von Zwischenlagern verpflichtet und die Deckungsvorsorge für AKW erhöht. Das AtG trat am 27.4.2002 in Kraft.

Die 2. Große Koalition von CDU/CSU und SPD hielt 2005 im Koalitionsvertrag hinsichtlich der Nutzung der Kernenergie zur Stromerzeugung fest, dass unterschiedliche Auffassungen bestehen und deshalb die zwischen Bundesregierung und Energieversorgungsunternehmen geschlossene Vereinbarung, die darin enthaltenen Verfahren sowie die dazu in der Novelle des Atomgesetzes getroffenen Regelungen nicht geändert würden. Aber die Atompolitik der Bundesregierung änderte sich, nachdem im November 2009 eine konservativ-liberale Koalition ins Amt kam. Die neue Bundesregierung legte im Herbst 2010 ein »Energiekonzept für eine umweltschonende, zuverlässige und bezahlbare Energieversorgung« vor. In Zukunft soll Deutschland seine Energieversorgung immer mehr aus erneuerbaren Quellen decken. Mit dem Argument, die Kernenergie sei eine »Brückentechnologie« auf diesem Weg, wurden die Laufzeiten der AKW um durchschnittlich zwölf Jahre verlängert - die Novelle des AtG trat am 1.1.2011 in Kraft. Ein wesentlicher Teil der zusätzlichen Gewinne aus der Laufzeitverlängerung sollte von den Betreibern an den Staat abgeführt werden. Dieser »Ausstieg aus dem Atomausstieg« führte nicht nur zum Protest der kommunalen Energiewirtschaft, sondern mobilisierte vor allem die Anti-AKW-Bewegung. Es kam zu sehr großen Demonstrationen gegen die Kernenergie.

Die Reaktorkatastrophe im März 2011 in Fukushima beförderte schließlich einen sehr breiten Anti-Atom-Konsens in Deutschland. Fast alle gesellschaftlichen Gruppen, Kirchen, Regierungs- und Oppositionsparteien, einigten sich auf die Forderung nach einem »schnellstmöglichen« Ausstieg. Sprachrohr dieses Konsenses war die Ethikkommission "Sichere Energieversorgung «, deren Bericht im Mai 2011 an die Bundesregierung übergeben wurde. Noch im März hatte die Bundesregierung zunächst ein dreimonatiges Moratorium für die ältesten Reaktoren verhängt. Im Juni/Juli 2011 beschlossen Bundeskabinett, Bundestag und Bundesrat einen beschleunigten Ausstieg aus der Kernenergie. Acht Reaktoren blieben abgeschaltet und die restlichen werden bis Ende 2022 stufenweise stillgelegt.

In der deutschen Atompolitik geht es seitdem vor allem um das Problem der Endlagerung des Atommülls. Im Juli 2013 wurde das Standortauswahlgesetz (StandAG) vom Deutschen Bundestag mit den Stimmen von CDU/CSU, SPD, FDP und Grünen verabschiedet. Die Kommission »Lagerung hoch radioaktiver Abfallstoffe«, legte im Juli 2016 ihren Abschlussbericht vor (Endlager-Kommission 2016). Die Novellierung des StandAG vom März 2017 legt auf der Grundlage der Empfehlungen der Kommission die Kriterien für die Auswahl möglicher Endlagerungs-Standorte, Regelungen für Beteiligungsverfahren und den Ablauf des Standortauswahlverfahrens fest, darüber hinaus wurde eine neue Institutionenstruktur etabliert. Mit dem Gesetz zur Neuordnung der Verantwortung in der kerntechnischen Entsorgung wurde Ende 2016 geregelt, dass für Stillegung, Rückbau und Verpackung der radioaktiven Abfälle weiterhin die Betreiber zuständig bleiben - allerdings ist der Bund für die Durchführung und Finanzierung der Zwischen- und Endlagerung verantwortlich. Ende September 2020 legte die Bundeseigene Gesellschaft für Endlagerung (BGE) den Zwischenbericht Teilgebiete vor. Der Zwischenbericht zeigt auf, welche Gebiete in Deutschland bei der Endlagersuche schon 
jetzt ausgeschlossen werden können und benennt diejenigen Gebiete, die im weiteren Verfahren näher untersucht werden - 90 sogenannte Teilgebiete (BGE 2020). Seit diesem Zeitpunkt gibt es potenziell Betroffene und die Maßnahmen zur Öffentlichkeitsbeteiligung müssen sich bewähren - und damit der »weichere« Staat.

\section{Schlussbetrachtungen}

Der Einstieg in die Nutzung der Atomenergie war und ist irreversibel; dies manifestiert sich insbesondere im Umgang mit den Ewigkeitslasten von rund $28.000 \mathrm{~m}^{3}$ hochradioaktiver Abfälle, die in Deutschland möglichst sicher eingelagert werden müssen. Doch der Umgang mit einer Vielzahl an Interessen und konkurrierenden Utopien verschiedener Akteure ist komplex und kompliziert (Häfner 2016: 10).

Die technische Utopie der »friedlichen Nutzung« der Kernenergie mit ihrem mehrstufigen Entwicklungsprogramm hat sich weltweit nicht erfüllt. Das heißt aber nicht, dass nicht beständig neue Atomtechnologien vorgestellt werden: seien es Small Modular Reactors, neue Reaktorlinien oder Ideen zu Transmutation. Die historische Liste technologischer Ansätze zur Entsorgung radioaktiver Substanzen ist lang: in den 1950er Jahren über die Kanalisation, die Versenkung des Materials in über 2.000 Metern Tiefe im Meer, die utopische Idee der Verbringung durch Raketen in das Weltall, die Präferenz geologischer Tiefenlager in Steinsalz ohne vorhergesehene Rückholung ab den 1960er Jahren, die bis in die 1980er Jahren favorisierte Wiederaufarbeitung, dann die direkte Endlagerung abgebrannter Brennelemente und die Idee eines internationalen Endlagers in den späten 1980er und 1990er Jahren.

Derzeit wird in der BRD nach dem StandAG der Standort für ein geologisches Tiefenlager mit oder ohne vorgesehener Rückholung in den Wirtsgesteinen Ton, Steinsalz oder Granit gesucht. Die Optionen der Langzeit-Oberflächenlagerung (für zunächst bis zu 100 Jahren wie in den Niederlanden) oder die durch die Endlager-Kommission vorgeschlagene Option der tiefen Bohrlöcher sind derzeit aus dem Prozess der Endlagerstandortsuche - und auch aus dem öffentlichen Diskurs - verschwunden. Der Pfad der Entsorgung wird auch heute vor allem staatlich (und nicht ökonomisch) gesteuert - sei es durch Behörden, Kommissionen, Gesetze oder die Forschungsförderung.

Vielleicht erfüllt sich die Prognose Edgar Salins für das Atomzeitalter erst in der Beendigung desselben: „Das Unwiderrufliche ist geschehen [...] Die Atomwelt lässt nicht viel Freiheitsraum und engt auch das klassische Feld der Demokratie bedenklich ein, aber auf der anderen Seite mag die Furcht vor dem Sturz in den Abgrund den aufbauwilligen Kräften helfen, eine neue Ordnung der Gesellschaft zu verwirklichen, die wieder menschen- und lebenswürdig ist.« (Salin 1955: 44)

Gegen erhebliche Proteste lässt sich ein Endlagerprojekt nicht realisieren. Nachdem der »harte « Atomstaat nicht erfolgreich war, agieren staatliche Institutionen derzeit relativ weich - und das Verfahren der Endlagerstandortsuche ist transparenter und partizipativer. So wurden im Rahmen des StandAG neue Wege der Öffentlichkeitsbeteiligung geschaffen, die sich nun bewähren müssen. Das Verfahren muss ein wirklich lernendes Verfahren sein, um neue Möglichkeiten der Öffentlichkeitsbeteiligung 
$\mathrm{zu}$ entwickeln und um Fehler der Vergangenheit nicht zu wiederholen (Häfner 2019: 53).

Doch dennoch ist es für staatliche Stellen verführerisch, einfach »durchzuregieren«. Die Aufgabe der Wissenschaft wird es sein, den Bereich im Diskurs offen zu halten, in welchem staatliche Institutionen die Bereitschaft zu Dialog und Beteiligung zeigen - die Debatte aber gleichzeitig auf ihre eigene Agenda verengen wollen. Aufgabe der Sozialwissenschaften ist es, solche soziotechnischen Dialoge immer wieder um gesellschaftliche und soziotechnische Dimensionen zu bereichern - die folgenden Texte in diesem Band sind dazu ein Beitrag.

\section{Literatur}

Anders, Günter (1956): Die Antiquiertheit des Menschen. Band I: Über die Seele im Zeitalter der zweiten industriellen Revolution, München: C. H. Beck.

Bundesgesellschaft für Endlagerung mbH (BGE) (2020): Zwischenbericht Teilgebiete gemäß $\$ 13$ StandAG. https://www.bge.de/fileadmin/user_upload/Standortsuche/ Wesentliche_Unterlagen/Zwischenbericht_Teilgebiete/Zwischenbericht_Teilgebiet e_barrierefrei.pdf, zuletzt geprüft am 29.10.2020.

Bloch, Ernst (1959): Das Prinzip Hoffnung, Frankfurt a.M.: Suhrkamp.

Blowers, Andrew (2017): The Legacy of Nuclear Power, New York: Routledge.

Brunnengräber, Achim (2019, 2. Überarbeitete Auflage): Ewigkeitslasten. Die »Endlagerung « radioaktiver Abfälle als soziales, politisches und wissenschaftliches Projekt, Baden-Baden: edition sigma in der Nomos Verlagsgesellschaft/zugleich: Schriftenreihe der Bundeszentrale für politische Bildung bpb, Band 10361, Bonn

BMU (1998): Nuclear Safety, in: Bericht der Regierung der Regierung über nukleare Sicherheit im Rahmen des Übereinkommens über nukleare Sicherheit für die erste Überprüfungssitzung im April 1999, Bonn.

Brand, Karl-Werner et al. (1986): Aufbruch in eine andere Gesellschaft. Neue soziale Bewegungen in der Bundesrepublik, (2. Auflage), Frankfurt a.M.: Campus.

Deutscher Bundestag (1974): Erste Fortschreibung des Energieprogramms der Bundesregierung, BT-Drucksache 7/2713, Bonn.

Deutscher Bundestag (1980): Zukünftige Kernenergiepolitik. Kriterien - Möglichkeiten - Empfehlungen, Bericht der Enquete-Kommission des Deutschen Bundestages Teil I + II, Bonn.

Endlager-Kommission (2016) Abschlussbericht der Kommission Lagerung hoch radioaktiver Abfallstoffe, K.-Drs. 268.

https://www.bundestag.de/resource/blob/434430/bb37b21b8e1e7e049ace5db6b2f94 9b2/drs_268-data.pdf, zuletzt geprüft am 11.11.2020.

Gleitsmann, Rolf-Jürgen/Oetzel, Günther (2012): Fortschrittsfeinde im Atomzeitalter? Protest und Innovationsmanagement am Beispiel der frühen Kernenergiepläne der Bundesrepublik Deutschland, Technikdiskurse. Karlsruher Studien zur Technikgeschichte, Band 5, Diepholz: GNT-Verlag. 
Graul, E. Heinz (1956): Sicherheits- und Schutzprobleme bei Reaktorprojekten und Umgang mit radioaktiven Isotopen. IV Radioaktive Abfälle, In: Atompraxis, Jahrgang 1955/56, Karlsruhe: Verlag G. Braun, 314-320.

Graul, E. Heinz (1957): Sicherheits- und Schutzprobleme bei Reaktorprojekten und Umgang mit radioaktiven Isotopen. VI Mitteilung: Versuch zur Beurteilung von Kernreaktorkatastrophen, In: Atompraxis, Jahrgang 1957, Karlsruhe: Verlag G. Braun, 468-476.

Häfner, Daniel (2016): Screening der Akteure im Bereich der Endlagerstandortsuche für radioaktive Reststoffe in der Bundesrepublik Deutschland, ENTRIA-Arbeitsbericht \#4. https://www.entria.de/fileadmin/entria/Dokumente/Arbeitsberichte/ENTRIAArbeitsbericht-04_Haefner_Akteursscreening.pdf, zuletzt geprüft am 4.5.2020.

Häfner, Daniel (2019): Partizipation rückwärts. Zur Aufarbeitung des Atomkonfliktes, in: Sommer, Jörg (2019): Kursbuch Bürgerbeteiligung \#3, S. 41-57.

Hennicke, Peter et al. (2000): Kernkraftwerksanalyse im Rahmen des Projekts: Bewertung eines Ausstiegs aus der Kernenergie aus klimapolitischer und volkswirtschaftlicher Sicht, Zusatzauftrag: Kraftwerks- und unternehmensscharfe Analyse, Wuppertal Institut für Klima, Umwelt und Energie, Öko-Institut, 27. Januar 2000.

Jungk, Robert (1977): Der Atomstaat. Vom Fortschritt in die Unmenschlichkeit, München: Kindler.

Keck, Otto (1984): Der Schnelle Brüter: Eine Fallstudie über Entscheidungsprozesse in der Großtechnik, Frankfurt/M, New York: Campus.

Klaus, Georg/Porst, Peter (1949): atomkraft - atomkrieg?, Berlin (Ost): Verlag Kultur und Fortschritt.

Künkel, H.A. (1956): Zum Problem der Beseitigung radioaktiver Abfälle, In: Atompraxis, Jahrgang 1955/56, Karlsruhe: Verlag G. Braun, 272-274.

Mez, Lutz (1979): »Bundesrepublik Deutschland - Der unaufhaltsame Aufstieg zur Atommacht«, In: Lutz Mez (Hg.): Der Atomkonflikt. Atomindustrie. Atompolitik und Anti-Atom-Bewegung im internationalen Vergleich, Berlin: Olle \& Wolter, 2953.

Mez, Lutz (1997): »Energiekonsens in Deutschland? Eine politikwissenschaftliche Analyse der Konsensgespräche - Voraussetzungen, Vorgeschichte, Verlauf und Nachgeplänkel«, In: Hans Günter Brauch (Hg.): Energiepolitik, Berlin etc.: Springer, 433448.

Mez, Lutz/Piening, Annette (2006): »Phasing-Out Nuclear Power Generation, in: Policies, Actors, Issues and Non-Issues«, In: Martin Jänicke \& Klaus Jacob (Eds.), Environmental Governance in Global Perspective. Neue Ansätze zur ökologischen Modernisierung, FFU Report 01-2006, 322-349. Berlin.

Mirow, Kurt Rudolf (1980), Das Atomgeschäft mit Brasilien: Ein Milliardenfiasko. Fran kfurta.M.,NewYork:Campus.

Prüß, Karsten, (1974) Kernforschungspolitik in der Bundesrepublik Deutschland, Frankfurt a.M.: Suhrkamp.

Radkau, Joachim (1983): Aufstieg und Krise der deutschen Atomwirtschaft: 1945-1975, verdrängte Alternativen in der Kerntechnik und der Ursprung der nuklearen Kontroverse, Reinbek: Rowohlt. 
Radkau, Joachim/Hahn, Lothar (2013): Aufstieg und Fall der deutschen Atomwirtschaft, München: Oekom.

Radkau, Joachim (2017): Geschichte der Zukunft. Prognosen, Visionen, Irrungen in Deutschland von 1945 bis heute, München: Carl Hanser Verlag.

Strauß, Franz Josef (1956): Rede anlässlich der Konstituierung der Deutschen Atomkommission vom 26. Januar 1956. https://www.fjs.de/fileadmin/user_upload/FJS/D ocuments/politiker/Atomkommission_Rede.pdf, zuletzt geprüft am 4.5.2020.

Stephany, Manfred (2005): Zur Geschichte der NUKEM: 1960 bis 1987, Norderstedt: books on demand. 